Short Communication

\title{
Lack of functional wolframin causes drop in plasmalemmal sodium-calcium exchanger type 1 expression at early stage in rat model of Wolfram syndrome
}

\author{
Simona Kurekova ${ }^{1}$, Mario Plaas ${ }^{2,3}$ and Michal Cagalinec ${ }^{4,5,6,7}$ \\ ${ }^{1}$ Department of Biophysics and Electrophysiology, Institute of Molecular Physiology and Genetics, Centre of Biosciences, \\ Slovak Academy of Sciences, Bratislava, Slovakia \\ ${ }^{2}$ Department of Physiology, Institute of Biomedicine and Translational Medicine, Faculty of Medicine, University of Tartu, \\ Tartu, Estonia \\ ${ }^{3}$ Laboratory Animal Centre, Institute of Biomedicine and Translational Medicine, Faculty of Medicine, University of Tartu, \\ Tartu, Estonia \\ ${ }^{4}$ Department of Cellular Cardiology, Institute of Experimental Endocrinology, Biomedical Research Center, Slovak Academy \\ of Sciences, Bratislava, Slovakia \\ ${ }^{5}$ Centre of Excellence for Advanced Materials Application, Slovak Academy of Sciences, Bratislava, Slovakia \\ ${ }^{6}$ Department of Neurophysiology and Neuropharmacology, Center for Physiology and Pharmacology, Medical University of \\ Vienna, Vienna, Austria \\ ${ }^{7}$ Department of Pharmacology, Institute of Biomedicine and Translational Medicine, Faculty of Medicine, University of \\ Tartu, Tartu, Estonia
}

\begin{abstract}
In previously introduced rat model of Wolfram syndrome, we have shown that in cardiac myocytes lacking functional wolframin protein the calcium transients and contractile response are significantly changed. Therefore, in this model, we evaluated protein and mRNA expression levels of following proteins involved in cardiac myocytes calcium homeostasis: the ryanodine receptor type 2 , calsequestrin type 2 , the junctophilin type 2 and plasmalemmal sodium-calcium exchanger type 1 (NCX1). For NCX1 we detected a significant decrease in expression both on protein and mRNA level. Thus, beyond its impact on endoplasmic reticulum stress, calcium, and mitochondria, wolframin influences processes in the myocyte plasma membrane.
\end{abstract}

Key words: Wolfram syndrome - Ryanodine receptor - Sodium-calcium exchanger - Calmodulin - Calsequestrin

Wolfram syndrome (WS; OMIM \#222300) is a rare hereditary disorder characterized by diabetes insipidus, early-onset diabetes mellitus, optical atrophy and deafness as cardinal symptoms, therefore named also as DIDMOAD (Wolfram 1938). WS is a prototype of endoplasmic reticulum (ER) disorder where the majority of the patient cases represent mutations of the wolframin protein (Wfs1) (Urano

Correspondence to: Michal Cagalinec, Department. of Cellular Cardiology, Institute of Experimental Endocrinology, Biomedical Research Center, Slovak Academy of Sciences, Dubravska cesta 9, 84505 Bratislava, Slovakia

E-mail: michal.cagalinec@savba.sk
2016). Wfs 1 harbours membrane of the ER and its function is not fully elucidated yet. What is known so far, the Wfs 1 is involved in the regulation of ER stress, mitochondrial functioning and is strongly involved in cell calcium metabolism. In HEK cells, Wfs 1 has been shown to positively modulate $\mathrm{Ca}^{2+}$ level in the ER by increasing the rate of $\mathrm{Ca}^{2+}$ uptake (Takei et al. 2006). Because it contains at least 9 transmembrane domains, it was also suggested that it forms a specific calcium channel (Osman et al. 2003). In line with the Wfs 1 calcium modulation feature, our previously published data have shown that cytosolic calcium signal in response to glutamate or $\mathrm{KCl}$ stimulation was twice lower in Wfs1 deficient neurons (Cagalinec et al. 2016). Analogical 
decrease of the cytosolic calcium release amplitude has been observed recently in WS patients' derived fibroblasts after stimulation with bradykinin (La Morgia et al. 2020) or histamine (Angebault et al. 2018). Moreover, Zatyka et al. (2015) have demonstrated that the Wfs 1 co-immunoprecipitates with sarcoplasmic-endoplasmic reticulum calcium ATPase (SERCA) and that it negatively regulates SERCA expression. Finally, dantrolene, a well-known inhibitor of ryanodine receptor (RyR) was able to prevent neuronal death in WS by inhibiting the ER calcium leakage to the cytosol (Lu et al. 2014). Wfs 1 is highly expressed in the brain and pancreas where the expression corresponds to the symptoms of the disease (Yamada et al. 2006). Moreover, a high expression level has been observed in the heart (Yamada et al. 2006) and the evidence about cardiac complications, although originally not recognized as the cardinal ones, is growing constantly: heart malformations and anterior pituitary dysfunction were recognized and participated in the morbidity and mortality of some WS patients (Medlej et al. 2004). In some WS patients sinus tachycardia, atrial or ventricular arrhythmias have been observed (Fabbri et al. 2005). In a study involving 23 Spanish patients with WS the heart disorders are mentioned as adjacent manifestations of WS (Esteban Bueno and Gomez Trujillo 2006). To study the WS in more detail, a mouse (Koks et al. 2009) and later a rat model of the $W f_{s} 1$ deficiency has been developed (Plaas et al. 2017). In the latter, the animals miss the exon 5 of the $W f_{s 1}\left(\mathrm{Wfs} 1^{-\mathrm{e} 5 /-\mathrm{e} 5}\right.$ ) and develop the diabetic phenotype at the age of nine months. Therefore, to study the impact of the invalidation of the Wfs 1 independently from the diabetic condition, in our recent paper (Cagalinec et al. 2019) we have been evaluating the calcium transients and contractility in the left ventricular myocytes freshly isolated from the hearts of four months old animals. We have found, that duration of calcium transients as well as duration of myocyte contractile response were significantly prolonged. In case of contractility, the amplitude of contraction was higher in Wfs1 invalidated animals. Moreover, we have shown, that these perturbations are not associated with the expression of the SERCA2 on the protein level. To explain the findings how exactly Wfs 1 impacts calcium transients (and thus contractility), in this work we have evaluated the protein and RNA expression levels of several key proteins involved in the cardiac myocytes calcium homeostasis, namely the main sarcoplasmic reticulum (SR) calcium release channel type 2 (ryanodine receptor type 2, RyR2), calsequestin 2 (CASQ2) - the SR lumen calcium buffering protein, plasmalemmal sodium-calcium exchanger type 1 (NCX1) and the cardiac-specific protein involved in tethering plasmalemmal L-type calcium channel and the RyR2 for calcium-induced calcium release, the junctophilin 2 (JPH2) in myocytes isolated from the left ventricle of the four months old Wfs1 $1^{-\mathrm{e} 5 /-\mathrm{e} 5}$ rats.
All experiments were carried out in compliance with the guidelines for the care and use of laboratory animals and approved by the State Veterinary and Food Administration of the Slovak Republic in accordance with the Order of the Slovak government No. 289/2003 (approval No. 1007/16-221 and 2498/18-221a). Left ventricular cardiomyocytes were isolated from four months old male $\mathrm{Wfs} 1^{\text {-e5/-e5 }}$ rats (Plaas et al. 2017) by a protocol described in Cagalinec et al. (2019). Briefly, after heparin and pentobarbital administration the heart was quickly fixed to the cannula of Langendorff's apparatus and perfused with the following solutions: calcium modified Tyrode's solution for $5 \mathrm{~min}$; calcium-free modified Tyrode's solution for $5 \mathrm{~min}$, and finally with Liberase TM enzyme solution (Roche Diagnostics, Germany, Cat. No. 05401127 001) for $8 \mathrm{~min}$. The left ventricle was then cut into small pieces and filtered through a nylon mesh $(100 \mu \mathrm{m})$. The filtrate was then centrifugated at $50 \times g$ for $1 \mathrm{~min}$, the supernatant was replaced with calcium-free modified Tyrodes solution with addition of $0.05 \mathrm{CaCl}_{2}$ and resuspended thus creating the first batch of isolated myocytes. In parallel, the remaining tissue was incubated in the enzyme solution with addition of $1 \%$ (w./v.) of bovine serum albumin (BSA) to attenuate the enzyme activity for $5 \mathrm{~min}$ and the process of filtering, centrifugation and incubation was repeated until the remaining tissue was difested completely, usually yielding 3 to 4 batches. The batch with the highest yield of the rodshaped myocytes was taken for experiments.

Total RNA from Wfs1 $1^{-\mathrm{e} 5 /-\mathrm{e} 5}$ and $\mathrm{Wfs} 1^{+/+}$cardiomyocytes was isolated using TRIzol Reagent Solution (Invitrogen, USA) according to the suppliers ' instructions. Complementary DNA (cDNA) was synthesized from $2000 \mathrm{ng}$ of total RNA using the High Capacity cDNA Reverse Transcription kit (Applied Biosystems, USA) and attached protocol in a total volume of $20 \mu \mathrm{l}$.

The qRT-PCR analysis was performed using the CFX96 Touch Real-Time PCR Detection System (Bio-Rad, CA, USA). Each PCR reaction was performed in a total volume of $20 \mu \mathrm{l}$ using a TaqMan ${ }^{\circledR}$ Gene Expression Assays for RT-PCR (Thermo Scientific, Germany). TaqMan Assay include primers for CASQ2 (rn00567508_m1), RyR2 (rn01470303_m1), JPH2 (rn01239933_m1), NCX1 (rn04338914_m1) or ACTB (rn00667869_m1). All primers are supplied by Thermo Scientific, Germany. Expression $\beta$-actin (ACTB) was used as a housekeeper. Each PCR reaction was performed in triplicate. PCR reactions were performed under following conditions: $2 \mathrm{~min}$ at $50^{\circ} \mathrm{C}$ and $20 \mathrm{~s}$ at $95^{\circ} \mathrm{C}$ followed by 40 cycles of the thermal cycling of $3 \mathrm{~s}$ at $95^{\circ} \mathrm{C}$ and $30 \mathrm{~s}$ at $60^{\circ} \mathrm{C}$ in a 96 -well reaction plate. The amplification curves were evaluated using CFX Manager software (BioRad, CA, USA). The $2^{-\Delta \Delta C t}$ method of Livak et al. was used to evaluate relative quantification (Livak and Schmittgen 2001). 
The ventricular cardiomyocytes were lyzed in Ripa buffer and assayed for the protein content by Lowry's method according to the protocol described in Cagalinec et al. (2019). $25 \mu \mathrm{g}$ or $50 \mu \mathrm{g}$ of protein from each cell lysates was separated by $10 \%$ or gradient (4-20\%, MiniProtean TGX Gels, Bio-Rad) SDS-PAGE and transferred to PVDF membrane. Membranes were blocked $2 \mathrm{~h}$ with $5 \%$ low-fat milk in PBST $(0,1 \%$ Tween-20 in phosphate buffered saline (PBS)) and then incubated with primary antibody overnight. Primary antibodies used were antiCASQ (1:1000, PA1-913, ThermoFisher), JPH2 (1:200, SC-377086, Santa Cruz), anti-NXC1 (1:1000, ab177952, Abcam), anti-RyR (1:1000, MA3-916, ThermoFisher) and anti-tubulin (1:1000, ab56676, AbCam). Membranes were washed next with PBST and then incubated with the secondary antibodies $1.5 \mathrm{~h}$ at room temperature: HRP anti-mouse antibody (1:2000, W4021, Promega) or HRP anti-rabbit antibody (1:2000, W4011, Promega). The bands were visualized with a chemiluminescence immunodetection system (Amersham Biosciences, USA). Images were analyzed with the ImageLab software (BioRad, USA) based on optic densitometric analysis of the bands. Background was subtracted by a local contrast.

The data are expressed as the mean \pm SEM. The differences between groups were compared using unpaired Student's $t$-test with the conservative assumption of unconsisted SD between groups. $p$-values of $<0.05$ were considered statistically significant. Data analysis was performed in GraphPad Prism 6.0 (GraphPad, USA).

First, we evaluated expression of main calcium release channel in the membrane of cardiac myocyte sarcoplasmic reticulum - the RyR2. For both cases, the protein and mRNA level, we did not observe any significant differences $(p=$ 0.113 and $p=0.440$ ). This may suggest, that there is a functional disturbance of the RyR2 kinetics rather than change in expression level as the calcium transient duration in the $\mathrm{Wfs}^{\mathrm{e}}{ }^{\mathrm{e} / \mathrm{ee} 5}$ myocytes was significantly prolonged (Cagalinec et al. 2019). The next protein evaluated was the main ER/SR lumen calcium buffering protein CASQ2. In this case, normalized protein level in the Wfs1 group was not significantly different $(p=0.176)$, similarly as for mRNA level $(p=0.205)$. Next, we tested the protein and mRNA level of JPH2, a protein tethering L-type calcium channels in the plasma membrane and the RyR2 located in the membrane of ER/SR. Here, there was any significant difference in normalized protein level $(p=0.093)$, which was consistent with the results found on mRNA level $(p=0.631)$. Intriguingly, statistical analysis of the expression levels of NCX1 revealed significant drop both at protein $(p=0.025)$ and mRNA $(p=0.014)$ levels. The data are summarized in the Fig. 1 for mRNA and in Fig. 2 for protein expression levels. To be able to explain how the loss-offunction Wfs1, localized in the membrane of ER/SR can lead to observed drop in expression of the plasmalemmal NCX1, the functional and spatial communication between the ER and plasmalemma has to be taken into consideration. As the expression level of the JPH2, a protein involved in functional bridging the two membranes in the dyads was not changed, we suggest either functional disturbance of JPH2 or some alternative mechanism how the invalidated integral protein of the ER may change the expression level of a plasmalemmal protein. In the latter, one possible explanation may be the involvement of Wfs1 in ER stress (Yamada et al. 2006; Fonseca et al. 2010; Cagalinec et al. 2016), associated with the unfolded protein response where Wfs1 may influence protein synthesis and folding of the plasmalemmal NCX1. Supporting this concept, trafficking of the amino acid transporter $\mathrm{B}^{0,+}$ (SLC6A14) to the plasma membrane involves an exclusive interaction with SEC24C for its exit from the ER (Kovalchuk et al. 2019), where the Wfs1 also may be a part of this pathway. More focused to the cardiac myocytes and ion transporters, group of Wang et al. (2018) has demonstrated recently, that protein MOG1 is involved in the regulation of the ER exit of cardiac sodium channel $\mathrm{Na}_{\mathrm{v}} 1.5$ in neonatal rat cardiomyocytes. Here we suggest the Wfs1 may act in an analogic way for the NCX1 or to be a molecular partner of the MOG1 involved in this pathway. This hypothesis is further supported by former work of Zatyka et al. (2008) who have demonstrated, that Wfs1 interacts with the $\beta 1$-subunit of the plasma membrane $\mathrm{Na}^{+} / \mathrm{K}^{+}$-ATPase, and this interaction may occur only during the maturation and assembly of $\mathrm{Na}^{+} / \mathrm{K}^{+}$-ATPase subunits in the membrane of ER. Moreover, in this work the protein expression level of $\mathrm{Na}^{+} / \mathrm{K}^{+}$-ATPase $\beta 1$-subunit is reduced in the condition of Wfs 1 mutations derived from WS patients. Therefore, a reduced $\mathrm{Na}^{+} / \mathrm{K}^{+}$-ATPase activity could be present in the Wfs $1^{-\mathrm{e} 5 /-\mathrm{e} 5}$ animals. Together with observed NCX1 downregulation it may indicate that two critical proteins essential for the course of refractory phase in the heart can be reduced. This fact may then, at least in

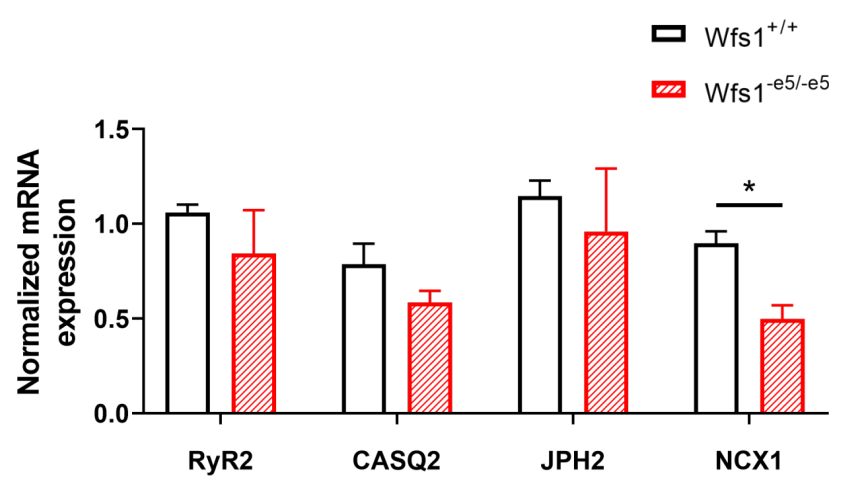

Figure 1. mRNA expression levels of selected proteins in left ventricular myocytes of Wfs $1^{-\mathrm{e} 5 /-\mathrm{e} 5}$ and $\mathrm{Wfs}^{+/+}$rats. Differences in gene expression of RyR2, CASQ2, JPH2 and NCX1 in ventricular cardiomyocytes Wfs1 $1^{-\mathrm{e} 5 /-\mathrm{e} 5}(n=3)$ and $\mathrm{Wfs}^{+/+}(n=3)$ animals. Expression ACTB was used as a loading control. ${ }^{\star} p<0.05$. 
A

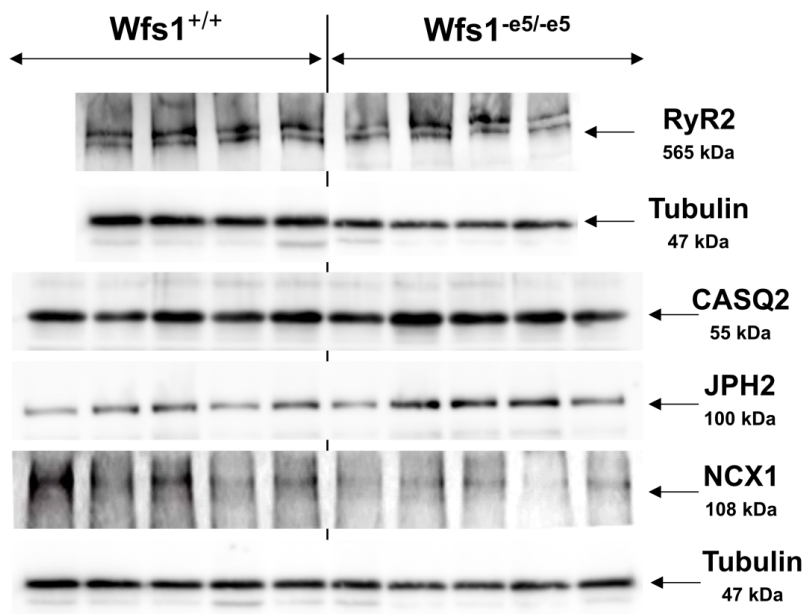

B
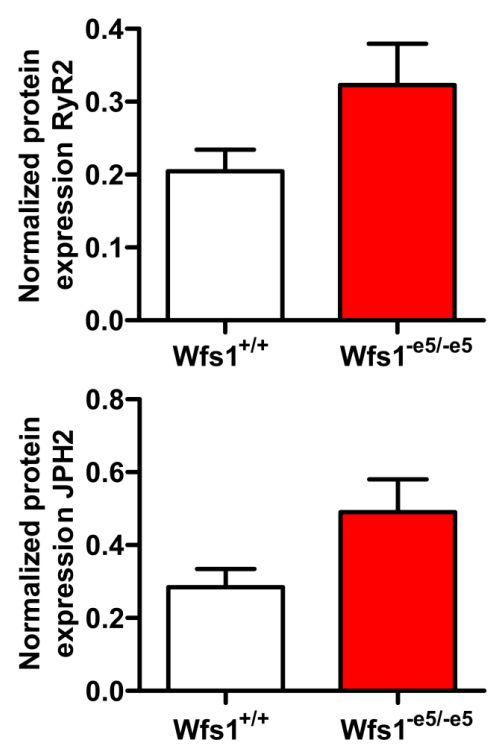
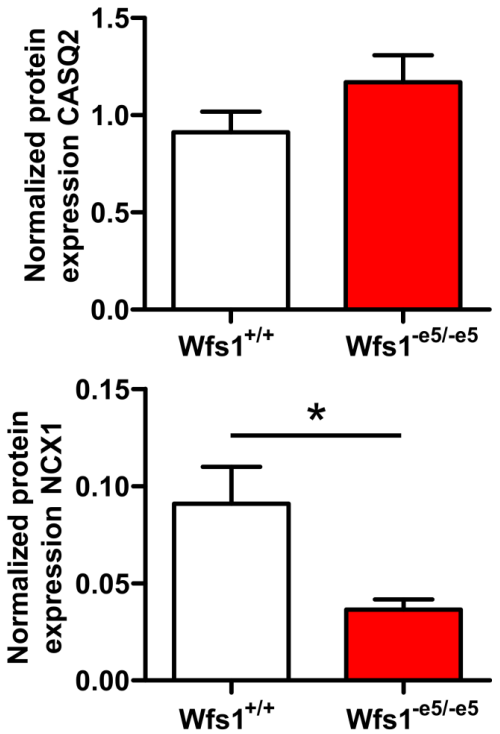

Figure 2. Protein expression levels of protein of interest in left ventricular myocytes of Wfs $1^{-\mathrm{e} 5 /-\mathrm{e} 5}$ and $\mathrm{Wfs}^{+/+}$rats. A. Western blot assay, Wfs1 $1^{-\mathrm{e} 5 /-\mathrm{e} 5}$ $(n=4-5)$ and $\mathrm{Wfs}^{+/+}(n=4-5)$. B. Quantification of the results shown in $\mathrm{A}$, the housekeeper protein tubulin was used as a loading control. The absence of functional Wfs 1 in myocytes resulted in a significantly reduced expression of NCX1, ${ }^{\star} p<0.05$. For RyR2, CASQ2 and JPH2 there was no significant difference in protein expression between $\mathrm{Wfs}^{-\mathrm{e} 5 /-\mathrm{e} 5}$ and control group.

part, explain the prolonged calcium transients duration and prolonged contraction duration in the $\mathrm{Wfs} 1^{-\mathrm{e} 5 /-\mathrm{e} 5}$ animals published recently (Cagalinec et al. 2019). Finally, as expression of the plasmalemmal NCX1 mRNA was significantly reduced concomitantly with the protein expression, this may indicate that the NCX1 protein reduction was a result of reduced NCX1 mRNA levels. Following this hypothesis, the deficiency of Wfs 1 is associated with increased levels of ER stress in pancreatic $\beta$-cells (Yamada et al. 2006) and neurons (Cagalinec et al. 2016) where the activation of major ER stress-response pathways and their downstream transcription factors may downregulate expression of several genes, including the NCX1 mRNA. However, in contrast to the pancreatic $\beta$-cells, the main ER stress pathways have not been detected in heart tissue of the Wfs1 deficient mice (Yamada et al. 2006), therefore to validate this pathway additional experiments are required for our experimental condition.
In conclusion, we show here for the first time the ER/SR located Wfs1 impacts beyond its effects on ER stress, mitochondrial functioning, and calcium homeostasis also the expression of myocyte plasmalemma proteins, in this case, the NCX1. These results shed more light on a better understanding of the Wfs 1 function and may help for more focused therapy of the WS and diabetic patients in the future.

Acknowledgements. This work was supported by grants APVV 150302; VEGA 2/0121/19, 2/0143/17, 2/0090/18, ITMS 26230120006 and PUT PSG471 from Estonian Research Council. We would like to thank to Alexandra Zahradnikova Jr for suggestions generally improving quality of the work, to Zdena Sulova with help to interpret the NCX1 Western blots and to Gizela Gajdosikova for excellent technical assistance with myocyte preparation. We also thank to the reviewer for the ideas to improve the discussion.

Conflict of interest. Authors declare no conflict of interest. 


\section{References}

Angebault C, Fauconnier J, Patergnani S, Rieusset J, Danese A, Affortit CA, Jagodzinska J, Mégy C, Quiles M, Cazevieille C, et al. (2018): ER-mitochondria cross-talk is regulated by the $\mathrm{Ca}(2+)$ sensor NCS1 and is impaired in Wolfram syndrome. Sci. Signal. 11, eaaq1380 https://doi.org/10.1126/scisignal.aaq1380

Cagalinec M, Liiv M, Hodurova Z, Hickey MA, Vaarmann A, Mandel M, Zeb A, Choubey V, Kuum M, Safiulina D, et al. (2016): Role of mitochondrial dynamics in neuronal development: Mechanism for Wolfram syndrome. PLoS Biol. 14, e1002511

https://doi.org/10.1371/journal.pbio.1002511

Cagalinec M, Zahradnikova A, Zahradnikova A, Jr., Kovacova D, Paulis L, Kurekova S, Hot'ka M, Pavelkova J, Plaas M, Novotova M, et al. (2019): Calcium signaling and contractility in cardiac myocyte of wolframin deficient rats. Front. Physiol. 10, 172 https://doi.org/10.3389/fphys.2019.00172

Esteban Bueno G, Gomez Trujillo FM (2006): Clinical manifestations and diagnostic delay in Wolframs syndrome. Rev. Clin. Esp. 206, 332-335 (in Spanish) https://doi.org/10.1157/13090481

Fabbri LP, Nucera M, Grippo A, Menicucci A, De Feo ML, Becchi C, Al Malyan M (2005): Wolfram syndrome. How much could knowledge challenge the fate? A case report. Med. Sci. Monit. 11, CS40-44

Fonseca SG, Ishigaki S, Oslowski CM, Lu S, Lipson KL, Ghosh R, Hayashi E, Ishihara H, Oka Y, Permutt MA, et al. (2010): Wolfram syndrome 1 gene negatively regulates ER stress signaling in rodent and human cells. J. Clin. Invest. 120, 744-755 https://doi.org/10.1172/JCI39678

Koks S, Soomets U, Paya-Cano JL, Fernandes C, Luuk H, Plaas M, Terasmaa A, Tillmann V, Noormets K, Vasar E, et al. (2009): Wfs1 gene deletion causes growth retardation in mice and interferes with the growth hormone pathway. Physiol. Genomics 37, 249-259 https://doi.org/10.1152/physiolgenomics.90407.2008

Kovalchuk V, Samluk L, Juraszek B, Jurkiewicz-Trzaska D, Sucic S, Freissmuth M, Nalecz KA (2019): Trafficking of the amino acid transporter $\mathrm{B}(0,+)$ (SLC6A14) to the plasma membrane involves an exclusive interaction with SEC24C for its exit from the endoplasmic reticulum. Biochim. Biophys. Acta 1866, 252-263 https://doi.org/10.1016/j.bbamcr.2018.11.005

La Morgia C, Maresca A, Amore G, Gramegna LL, Carbonelli M, Scimonelli E, Danese A, Patergnani S, Caporali L, Tagliavini F, Del Dotto V, et al. (2020): Calcium mishandling in absence of primary mitochondrial dysfunction drives cellular pathology in Wolfram Syndrome. Sci. Rep. 10, 4785 https://doi.org/10.1038/s41598-020-61735-3

Livak KJ, Schmittgen TD (2001): Analysis of relative gene expression data using real-time quantitative PCR and the 2(T)(-Delta Delta C) method. Methods 25, 402-408 https://doi.org/10.1006/meth.2001.1262

Lu S, Kanekura K, Hara T, Mahadevan J, Spears LD, Oslowski CM, Martinez R, Yamazaki-Inoue M, Toyoda M, Neilson A, et al.
(2014): A calcium-dependent protease as a potential therapeutic target for Wolfram syndrome. Proc. Natl. Acad. Sci. USA 111, E5292-5301

https://doi.org/10.1073/pnas.1421055111

Medlej R, Wasson J, Baz P, Azar S, Salti I, Loiselet J, Permutt A, Halaby G (2004): Diabetes mellitus and optic atrophy: a study of Wolfram syndrome in the Lebanese population. J. Clin. Endocrinol. Metab. 89, 1656-1661 https://doi.org/10.1210/jc.2002-030015

Osman AA, Saito M, Makepeace C, Permutt MA, Schlesinger P, Mueckler M (2003): Wolframin expression induces novel ion channel activity in endoplasmic reticulum membranes and increases intracellular calcium. J. Biol. Chem. 278, 52755-52762 https://doi.org/10.1074/jbc.M310331200

Plaas M, Seppa K, Reimets R, Jagomae T, Toots M, Koppel T, Vallisoo T, Nigul M, Heinla I, Meier R, et al. (2017): Wfs1deficient rats develop primary symptoms of Wolfram syndrome: insulin-dependent diabetes, optic nerve atrophy and medullary degeneration. Sci. Rep. 7, 10220 https://doi.org/10.1038/s41598-017-09392-x

Takei D, Ishihara H, Yamaguchi S, Yamada T, Tamura A, Katagiri H, Maruyama Y, Oka Y (2006): WFS1 protein modulates the free $\mathrm{Ca}(2+)$ concentration in the endoplasmic reticulum. FEBS Lett. 580, 5635-5640 https://doi.org/10.1016/j.febslet.2006.09.007

Urano F (2016): Wolfram syndrome: diagnosis, management, and treatment. Curr. Diab. Rep. 16, 6 https://doi.org/10.1007/s11892-015-0702-6

Wang Z, Yu G, Liu Y, Liu S, Aridor M, Huang Y, Hu Y, Wang L, Li S, Xiong H, et al. (2018): Small GTPases SAR1A and SAR1B regulate the trafficking of the cardiac sodium channel Nav1.5. Biochim. Biophys. Acta 1864, 3672-3684 https://doi.org/10.1016/j.bbadis.2018.09.003

Wolfram DJ, Wagener HP (1938): Diabetes mellitus and simple optic atrophy among siblings: report of four cases. Mayo Clin. Proc. 13, 715-718

Yamada T, Ishihara $\mathrm{H}$, Tamura A, Takahashi R, Yamaguchi $\mathrm{S}$, Takei D, Tokita A, Satake C, Tashiro F, Katagiri H, et al. (2006): WFS1-deficiency increases endoplasmic reticulum stress, impairs cell cycle progression and triggers the apoptotic pathway specifically in pancreatic beta-cells. Hum. Mol. Genet. 15, 1600-1609 https://doi.org/10.1093/hmg/ddl081

Zatyka M, Da Silva Xavier G, Bellomo EA, Leadbeater W, Astuti D, Smith J, Michelangeli F, Rutter GA, Barrett TG (2015): Sarco (endo) plasmic reticulum ATPase is a molecular partner of Wolfram syndrome 1 protein, which negatively regulates its expression. Hum. Mol. Genet. 24, 814-827 https://doi.org/10.1093/hmg/ddu499

Zatyka M, Ricketts C, da Silva Xavier G, Minton J, Fenton S, Hofmann-Thiel S, Rutter GA, Barrett TG (2008): Sodium-potassium ATPase 1 subunit is a molecular partner of Wolframin, an endoplasmic reticulum protein involved in ER stress. Hum. Mol. Genet. 17, 190-200 https://doi.org/10.1093/hmg/ddm296

Received: March 12, 2020

Final version accepted: May 11, 2020 